\title{
Antenatal Diagnosis of Renal and Excretory Tract Abnormalities: A Report of 36 Cases and Review of the Literature
}

\author{
Hekmat Chaara $^{1}$, Imane Attar ${ }^{1 *}$, Sofia Jayi ${ }^{1}$, Fatima-Zahra Fdili Alaoui ${ }^{1}$, Moulay Abdelilah Melhouf ${ }^{1}$ \\ ${ }^{1}$ Department of Gynecology and Obstetrics Ii, Chu Hassan Ii Fes, Morocco
}

DOI: $10.36348 /$ sijog.2021.v04i04.007

| Received: 07.03.2021 | Accepted: 06.04.2021 | Published: 17.04.2021

*Corresponding author: Imane Attar

Abstract

Common fetal urinary tract abnormalities encompass a complex spectrum of abnormalities grouped under the name CAKUT, which can be detected antenatal by ultrasound with an incidence of 1 to 4 in 1,000 pregnancies. As such, they represent 15 to $20 \%$ of all birth defects. They remain responsible for 30 to $50 \%$ of cases of end-stage renal failure, so it is crucial to have an early diagnosis and medical or surgical management in order to minimize renal damage and to avoid or delay a possible renal failure. Through a study carried out within our structure comprising 36 cases, we will recall the most frequent fetal anomalies of the kidneys and urinary tract by specifying the ultrasound modalities and the prognosis of these various malformations as well as the recommendations for pre and postnatal management.

Keywords: Morphological diagnosis, 1st trimester, prognosis, oligoamnios, associated malformation.

Copyright (C) 2021 The Author(s): This is an open-access article distributed under the terms of the Creative Commons Attribution 4.0 International License (CC BY-NC 4.0) which permits unrestricted use, distribution, and reproduction in any medium for non-commercial use provided the original author and source are credited.

\section{INTRODUCTION}

The introduction of routine ultrasound examination improved the detection rate of urinary tract abnormalities (from 0.1 to $2.3 \%$ ) which accounts for $20 \%$ of all fetal congenital anomalies [1, 2]. Common fetal urinary tract abnormalities encompass a complex spectrum of abnormalities grouped under the name CAKUT responsible for $40 \%$ of end-stage renal insufficiency, thus constituting a social and economic burden for parents and society [3, 4].

The development of the fetal urinary tract is a complex process, it is associated with a risk of birth defects with etiopathogenesis which still unknown. Common signs of urinary system abnormalities during the prenatal period are: oligohydramnios and changes in renal morphology, ureter or bladder either isolated or accompanied by extra-renal abnormalities [2].

The antenatal evaluation of the prognosis is the main objective of the obstetrician, knowing that this prognosis depends on several factors [3].

Through our work and a review of the literature we will try:
- To show the importance of antenatal ultrasound in the detection of urinary abnormalities as well as its relevance in the etiological diagnosis.

- To evaluate the prognostic factors for each malformation.

\section{MATERIALS}

A cross-sectional observational study was designed to examine 36 records of all women's who underwent an ultrasound and in whom an abnormality of the urinary tract was suspected, at the department of Gynaecology and Obstetrics II of the CHU Hassan II of Fez, from January 12011 to March 31, 2017. Data were collected from clinical records and ultrasound reports. The clinical and paraclinical parameters studied are:

- Epidemiological: maternal age, consanguinity, personal or family history, fetal sex.

- Details of the antenatal diagnosis: Age of diagnosis, biometry and amniotic fluid, morphology of the urogenital system, diagnoses suspected by ultrasound, topographic distribution, associated extra-renal malformations and syndromic approach.

- The outcome of pregnancy and the term of childbirth.

- The Apgar and the Weight at birth. 
The follow-up of the new-borns was in the short and medium term based on the results of their renal functions, the paraclinical explorations carried out and surgical interventions performed.

\section{RESULTS \\ Epidemiology}

36 cases of CAKUT were detected. The annual incidence has increased since the introduction of the antenatal diagnosis unit with an average of 6.3 new cases per year. The mean maternal age was $27.42 \pm$ 6.72 years, the majority of affected fetus were male $(66.67 \%)$. $25 \%$ of the fetuses were born from a consanguineous marriage. In our series, only $5.56 \%$ of our patients had familial history of chromosomal disease like Down syndrome, as was noted in a single woman a family history of end-stage renal failure (Table-1).

\section{Details of antenatal diagnosis Gestational age at diagnosis}

The 2nd and 3rd trimester ultrasound was performed respectively in $69.44 \%$ and $63.89 \%$ of cases. However, only $16.67 \%$ of cases benefited from a $1 \mathrm{st}$ trimester ultrasound, among which were observed: two cases of Autosomal recessive polycystic kidney disease and a single case of Meckel Grüber syndrome (Table2). The average age of diagnosis was 25.5 weeks.

\section{Biometrics and amniotic fluid}

$25 \%$ of our fetuses had Intrauterine growth restriction (IUGR). Among them, four had unilateral Multicystic dysplastic kidney (MCDK) and the other four had bilateral MCDK and one with bilateral renal agenesis. Unilateral kidney involvement can not explain the IUGR, it is necessary to seek a context to which the RCIU could be attached. 3/4 of the unilateral cases with IUGR in our series had associated malformations (Table-3).

An abnormality of the amniotic fluid is observed in $63.89 \%$ of our patients. $30.56 \%$ of cases had amniotic fluid of normal amount, while in 2 cases hydramnios was noted in mothers who had diabetes (Diagram-1).

\section{Morphology of the urogenital system}

The morphological study of the urogenital system was distributed as follows: renal involvement was $69 \%$, of which the structural anomaly remains the most common with a percentage of $55 \%$. An anomaly of the fetal upper excretory pathway was noted in $52.78 \%$ predominated by Ureteral dilation in $27.78 \%$ of cases, while $58 \%$ of cases had bladder abnormalities of which $36.11 \%$ of them the bladder was not visualized with a decrease in the amount of amniotic fluid of varying degree (Table-4).

Ultrasound suspected diagnoses and their topographies (Diagram-2)
- MCDK was the most frequently diagnosed fetal abnormality $(44.44 \%)$. in $62.50 \%$ of cases were bilateral and in the unilateral form a clear left predominance was noted (Figure 3A).

- Posterior urethral valves (PUV) were suspected in eight fetuses in our series (22.22\%) (Figure 3 B / C).

- Renal agenesis was observed in five fetuses (13.89\%), of which $80 \%$ were bilateral (Figure 3: D, E, F, G, H).

- Autosomal recessive polycystic kidney disease (ARKD) was suspected in four cases $(11.11 \%)$. the diagnosis was made on the basis of: the ultrasound aspect very evocative and the very early revelation (in two cases), a family history of renal pathology (in one case), the context of consanguinity (in two cases) (Figure-4).

- Four cases of Ureteropelvic junction (UPJ) were suspected $(11.11 \%)$, of which two cases were bilateral and two unilateral left cases. Both kidneys were dysplastic in a single case of bilateral SJPU, whereas in the other cases there was pyelocaliceal dilatation with preservation of corticomedullary differentiation (Figure-5C).

- Three cases of duplicated collecting system were suspected $(8.33 \%)$. All three cases were left unilateral (Figure 5A, B).

- Isolated fetal pyelectasis observed in two cases with a percentage of $5.56 \%$ with a predominance on the left side.

Associated extra-renal malformations and syndromes: according to all the cases studied and all the associated malformations (heart malformations $25 \%$, cerebral malformations $22 \%$, etc.), we were able to identify some syndromes: 2 cases of Meckel Grüber syndrome (diagnosed at $15 \mathrm{WA}+5$ days for the first one / 21 weeks $+2 \mathrm{~J}$ for the second) and Prune-Belly syndrome (diagnosed at $21 \mathrm{NW}+6 \mathrm{D}$ ).

\section{Pregnancy's outcomes, term of delivery and postpartum course:}

In $52.78 \%$ of pregnancies were a full-term pregnancy, $16.67 \%$ of pregnancies were Preterm birth, for seven pregnancies (19.44\%) there was intrauterine death (IUD). Medical abortion was carried out in three cases $(8.33 \%$ of cases) including 2 cases of Meckel Grüber Syndrome and the 3rd one was a bilateral MCDK associated with an anamnios and cervical cystic lymphangioma. Finally, only one pregnancy was lost to follow-up.

Of the five pre-term baby's, three died during the neonatal period: the first, carrying a MCDK associated with a duplicated collecting system, he was hospitalized in paediatric intensive care and neonates for respiratory distress and renal failure then died after 23 days; the second presented with bilateral MCDK which died after 10 hours of life; the 3 rd had polycystic 
Hekmat Chaara et al; Sch Int J Obstet Gynec, Apr. 2021; 4(4): 114-123

kidney disease who died after 1 day of life. The evolution was good for the other babies, 1 of them had a left MCDK with a duplicated collecting system and the second one had a PUV without dysplasia. For the 19 completed pregnancies there were 7 neonatal deaths and 12 survivors (Figures-1).

Table-1: Epidemiological characteristics of the studied population

\begin{tabular}{|c|c|c|c|}
\hline \multicolumn{4}{|c|}{ Epidemiological parameters } \\
\hline \multicolumn{2}{|c|}{ Maternal age } & \multicolumn{2}{|l|}{$27.42(6.72)$ years } \\
\hline \multicolumn{2}{|c|}{ Parity } & \multicolumn{2}{|l|}{$3.6(2.53)$} \\
\hline \multirow{2}{*}{\multicolumn{2}{|c|}{ Consanguinity : }} & \multirow[t]{2}{*}{$25 \%$} & $67.67 \%$ (1 degree $)$ \\
\hline & & & $33.33 \%$ (2 degree) \\
\hline \multirow[t]{8}{*}{ ATCD } & \multirow[t]{6}{*}{ Staff } & Miscarriage & $22 \%$ \\
\hline & & MFIU & $8.34 \%$ \\
\hline & & Neonatal death & $5.36 \%$ \\
\hline & & Diabetes & $13.89 \%$ \\
\hline & & Hyperthyroidism & $2.78 \%$ \\
\hline & & Plant catch (funegreg) & $2.78 \%$ \\
\hline & \multirow[t]{2}{*}{ Family } & ATCD of T21 & $5.56 \%$ \\
\hline & & Renal failure & $2.7 \%$ \\
\hline \multirow{3}{*}{\multicolumn{2}{|c|}{ FETAL SEX }} & MALE & $66.67 \%$ \\
\hline & & FEMININE & $30.56 \%$ \\
\hline & & UNDETERMINED & $2.78 \%$ \\
\hline
\end{tabular}

Table-2: Breakdown of suspected abnormalities in the 1st quarter

\begin{tabular}{|l|l|l|}
\hline Ultrasound appearance & Number of cases & Percentages \\
\hline Normal & $\mathbf{0 3}$ & $\mathbf{5 0 \%}$ \\
\hline Autosomal recessive polycystic kidney disease & $\mathbf{0 2}$ & $\mathbf{3 3 . 3 3 \%}$ \\
\hline Meckel Cruber Syndrome & $\mathbf{0 1}$ & $\mathbf{1 6 . 6 7 \%}$ \\
\hline Total & $\mathbf{0 6}$ & $\mathbf{1 0 0 \%}$ \\
\hline
\end{tabular}

Table-3: Context of intrauterine growth retardation for unilateral cases

\begin{tabular}{|l|l|}
\hline Context of IURR for unilateral cases & Number of Cases \\
\hline Brain and heart malformation & 1 \\
\hline Brain malformation & 1 \\
\hline Spinal dysgraphia and clubfoot & 1 \\
\hline Imperforate anus and spina & 1 \\
\hline
\end{tabular}

Table-4: Breakdown of cases according to the level of impairment

\begin{tabular}{|c|c|c|}
\hline \multicolumn{2}{|l|}{ Topography } & Rates \\
\hline \multirow[t]{3}{*}{ Kidney } & Structure & $55.55 \%$ \\
\hline & Number & $13.89 \%$ \\
\hline & Normal & $30.56 \%$ \\
\hline \multirow[t]{5}{*}{ Upper excretory pathway } & Pyelectasis & $16.69 \%$ \\
\hline & CPD & $25 \%$ \\
\hline & Ureteral dilation & $27.75 \%$ \\
\hline & Duplicate ureter & $2.78 \%$ \\
\hline & Normal & $27.78 \%$ \\
\hline \multirow[t]{3}{*}{ Bladder } & Megacyst & $19.44 \%$ \\
\hline & Bladder not seen & $38.89 \%$ \\
\hline & Normal bladder & $41.67 \%$ \\
\hline
\end{tabular}




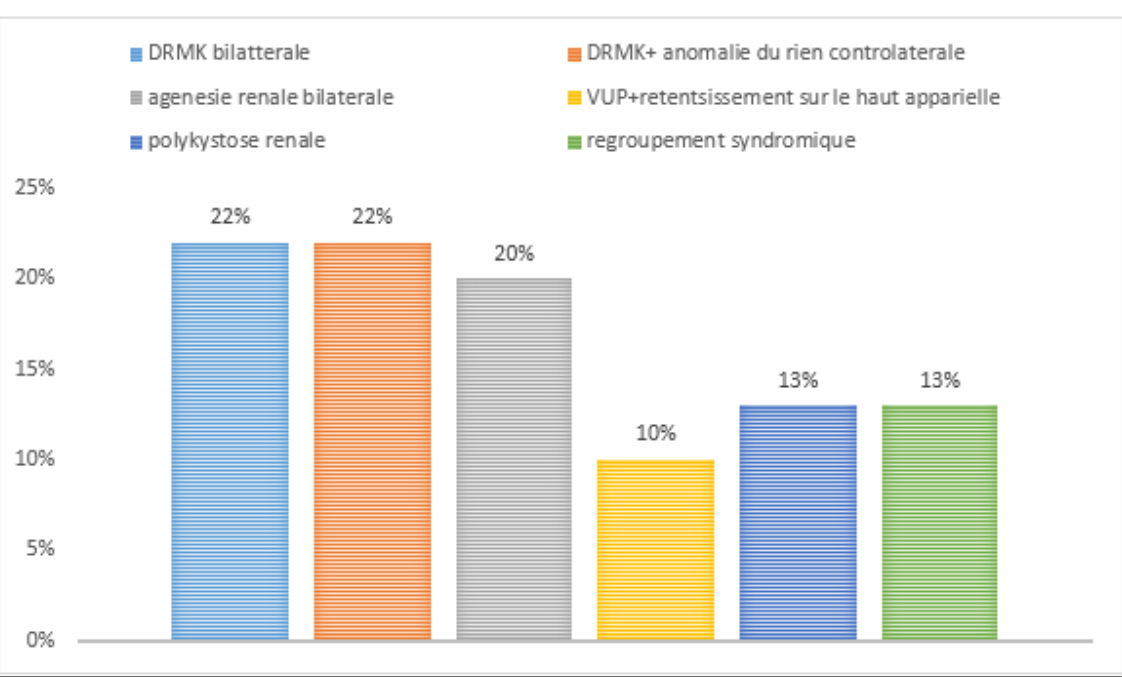

Diagram-1: Distribution of cases with oligo-anamnios according to the type of abnormality

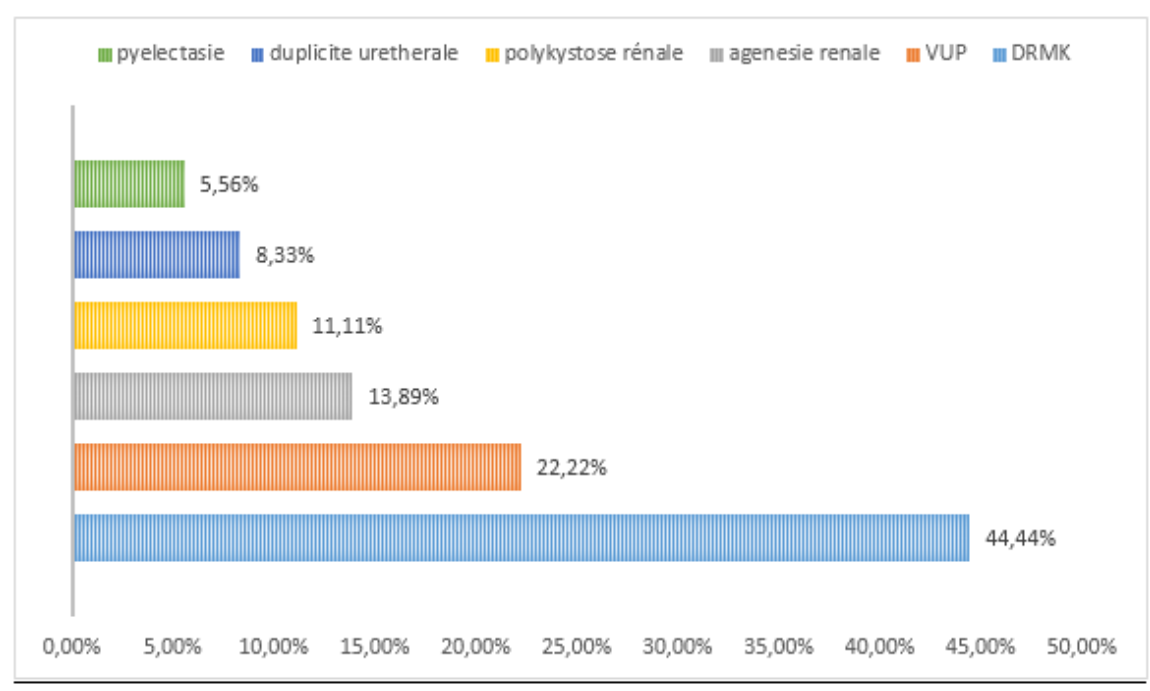

Diagram-2: Ultrasound suspected diagnoses

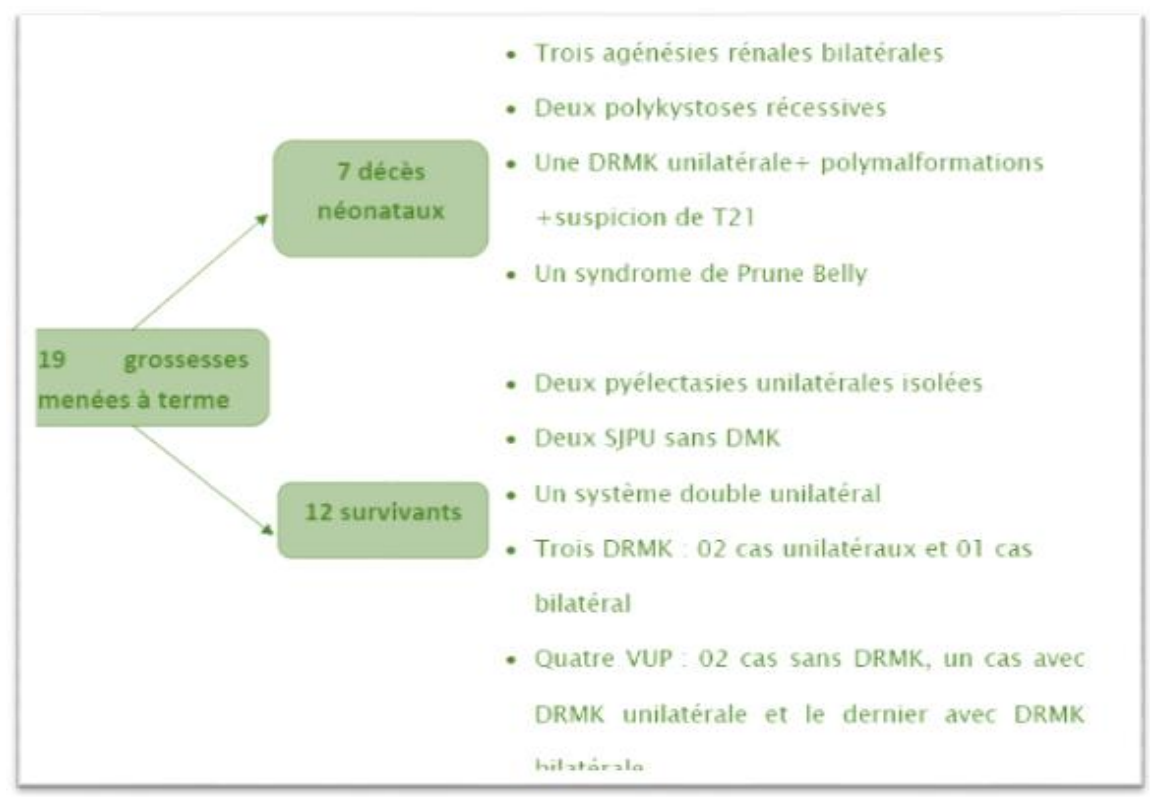

Fig-1: Outcome of completed pregnancies 

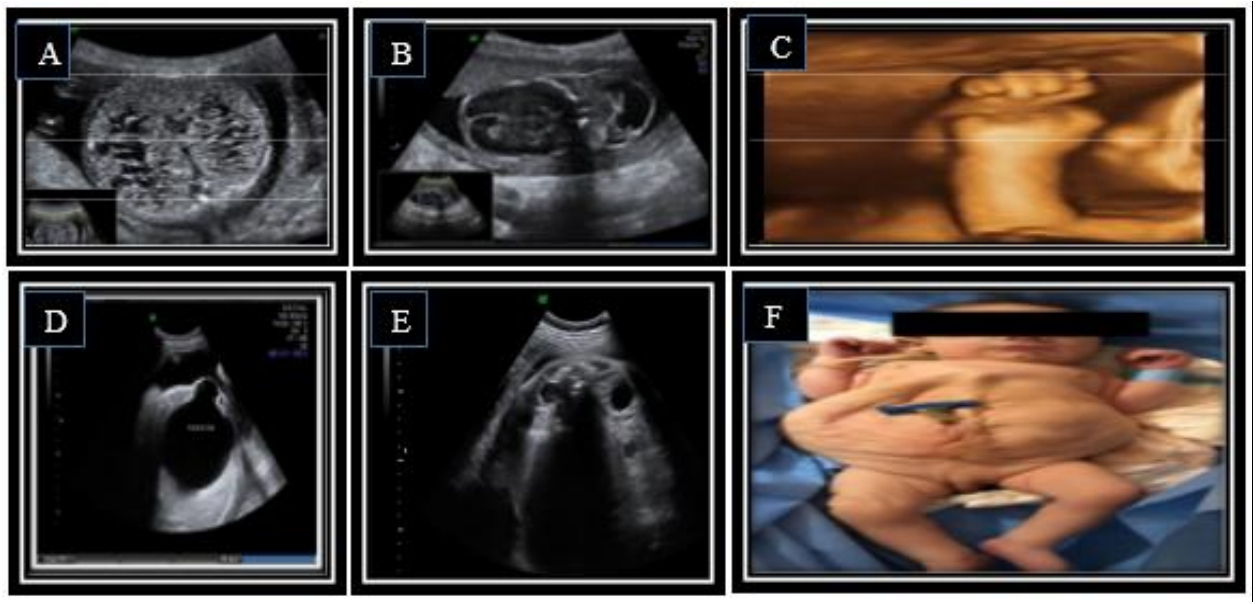

Fig-2: (A) Large dysplastic kidney with medullary cysts (B) Occipital encephalocele (C) Ultrasound reconstruction showing: Meckel Grüber syndrome (D) Huge megacystis (E) Pyelocaliceal dilation (F) Clinical appearance at birth with flaccid abdominal wall and bilateral cryptorchidism: Prune Belly syndrome
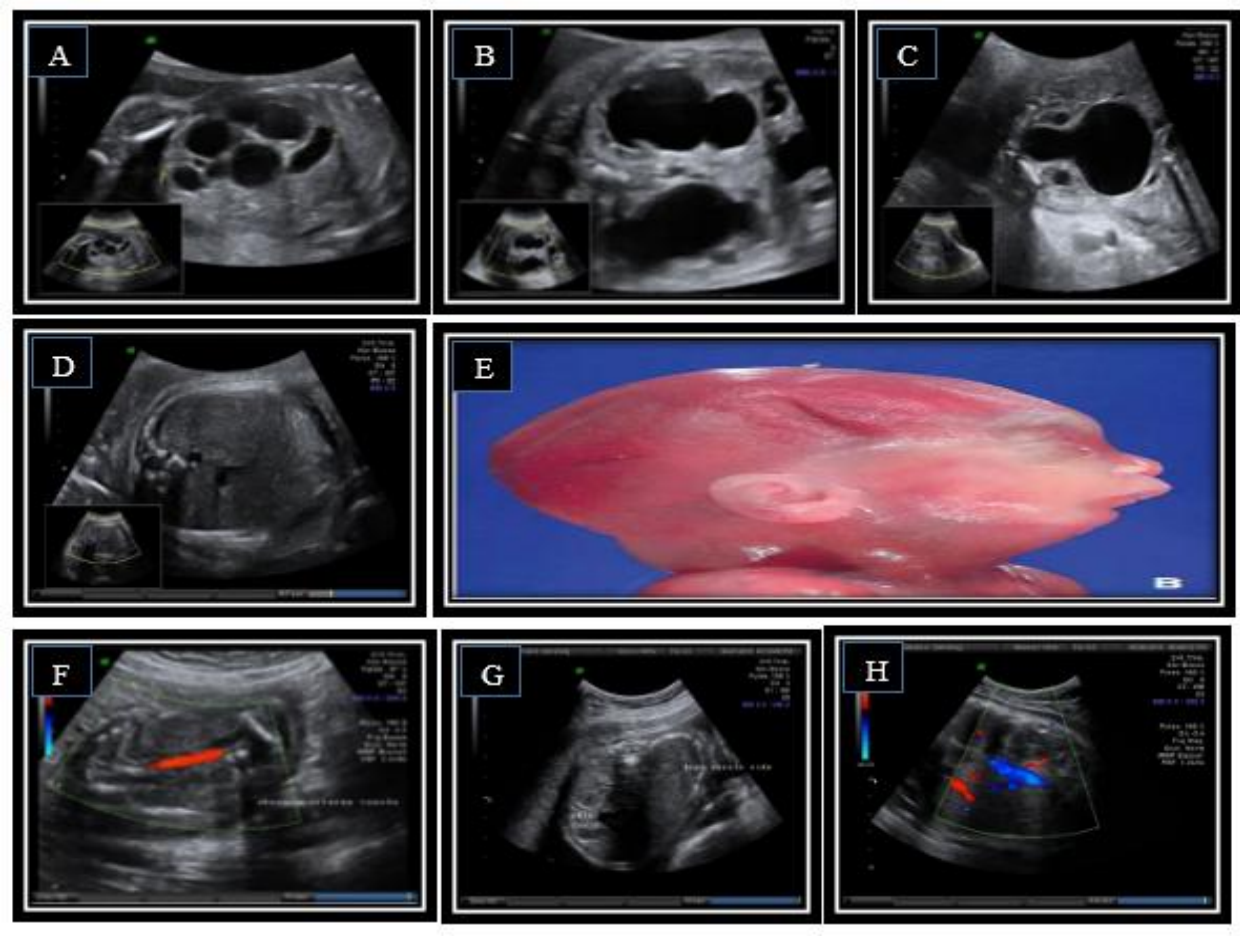

Fig-3: (A) Left MCDK (B) Bilateral pyelocaliceal dilation (C) Megacystis with bladder recess on PUV (D) absence of bilateral kidney visualization (E) Postnatal Potter's facies on bilateral renal agenesis (F) absence of renal arteries on color doppler. (G) Single left kidney with empty right renal compartment $(\mathrm{H})$ absence of the right renal artery on Doppler
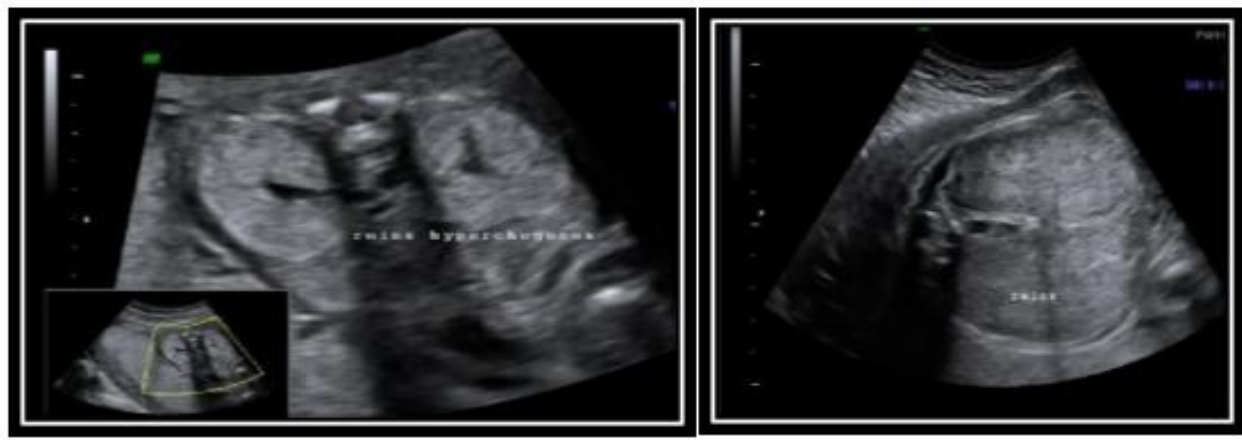

Fig-4: Recessive polycystic kidney disease: Large hyperechoic kidneys without cortico-medullary differentiation 

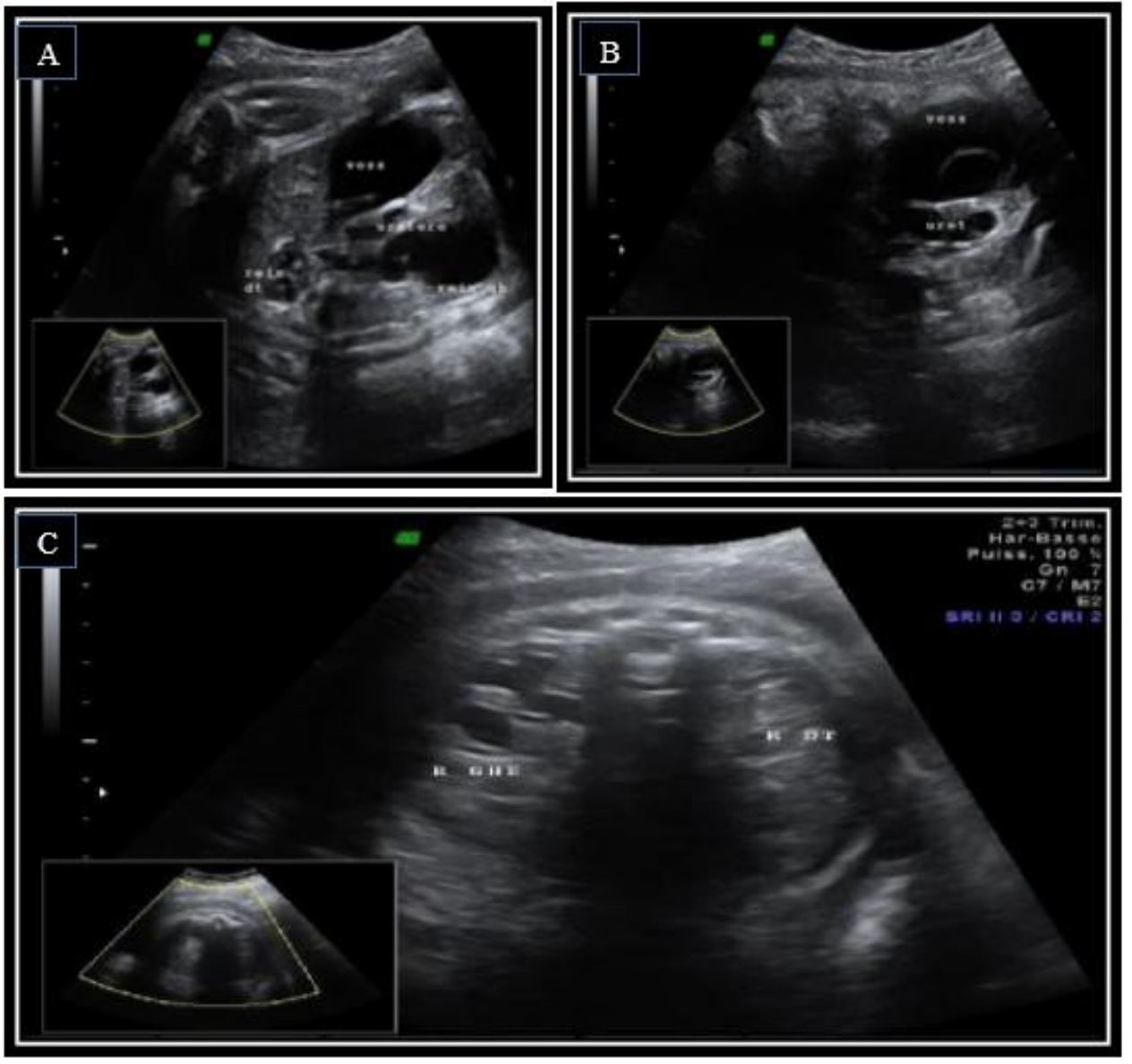

Fig-5: (A, B) Appearance of double pyelon with intravesical ureterocoele (C) Left pyelocaliceal dilation suggesting a junction syndrome

\section{DISCUSSION}

Congenital anomalies of the kidneys and urinary tract (CAKUT) are among the fetal congenital anomalies most commonly diagnosed on the prenatal period $(20-30 \%)$ with a sex ratio of $2.18[5,6]$. This is consistent with our study which collected 36 cases of CAKUT out of 180 cases of all malformations detected (20\%) tend to develop more often in men then in women $(66 \%)$. But they remain the cause of end-stage renal failure in 30 to $50 \%$ [4].

Its pathogenesis is not clear; they can be seen as sporadic cases or in a family history context, as they can be isolated or be a part of a multi organic defect process [7-10]. Therefore, Therefore, the exploration of extrarenal manifestations and the search for an underlying family history to establish a fetal prognosis seems mandatory. Our work consolidates these findings through a detailed morphological analysis which made it possible to detect certain associated extra-renal malformations, some of which belonged to a syndromic disease such as Meckel Grüber's syndrome (Figure 2A / B / C) and the Prune Belly Syndrome (Figure 2D, E and F).
Several factors were involved in the genesis of CAKUT. Genetic participation has been mentioned in several studies which makes it essential to research the notion of consanguinity which was found in $25 \%$ in our series similar to that reported by Shalaby et al., (24\%) but lower than that of Bondagji Jeddah-Saudi Arabia $(40 \%)[5,6]$. The search for personal or family history of fetal malformations or family history of renal pathologies can also point us towards a genetic origin [11], these factors are present respectively in our study with rates of $5.56 \%$ and $2.78 \%$. The factor most implicated in the occurrence of urinary tract malformations is gestational or pre-existing diabetes, $13.89 \%$ of the women in our series had diabetes at the time of diagnosis. [12]

Most studies suggest that the second trimester is the ideal time for the diagnosis of CAKUT, which agrees with our results with an average age of diagnosis of 25 WA $[15,16]$. However, certain familial pathologies can be detected in the first trimester, in our series of 6 patients who underwent a first trimester ultrasound, two cases had autosomal recessive polycystic kidney disease and only one case of Meckel Grüber syndrome. 
The prenatal diagnosis of uropathies and nephropathies includes the evaluation of the renal function of the fetus, as well as the echo-anatomical evaluation of the urinary system and the projection of the results obtained on the postnatal outcome. Fetal renal function is closely related to the amount of amniotic fluid considered to be a factor of poor prognosis when it is reduced, in this case any abnormality of the amniotic fluid requires a detailed analysis of the urinary tract $[9,16]$. All the published studies have confirmed a mortality that can go up to $100 \%$ in cases of oligoamnios, especially before 24 weeks. However, some authors demonstrate that fetal natriuresis and the Beta 2 Microglobulin dosage are not sufficiently reliable examinations for the precise assessment of routine renal function.

The echo-anatomical evaluation of the urinary tract is a codified and dynamic examination over time. In our series, we followed certain Semiological value which enabled us to detect the following anomalies:

\section{a. Multicystic dysplastic kidney}

MCDK is a major cause of end stage renal disease [4]. Its diagnosis is mainly possible by ultrasound from the 15th week of gestation with an average of 21 to 35 weeks WA, [17, 18]. The ultrasound description of MCDK at a sensitivity close to $100 \%$ with a semiology that differs depending on the underlying etiology, It can have segmental distribution patterns with bilateral or unilateral renal involvement, when it is unilateral, an associated anomaly of the contralateral kidney (like a vesicoureteral reflux or Ureteropelvic junction syndrome) should be carefully looked for and the morphological study should also focus on the extra-renal organs [19-21]. Once the diagnosis has been made, antenatal ultrasound monitoring is necessary in order to judge the evolving profile of MCDK [22]; As for the prognosis, renal dysplasia is recognized as being fatal in its bilateral form with a better prognosis for the unilateral form.

In our study, MCDK constituted the major part of the pathologies found (44.44\% of cases) and $68.75 \%$ of dysplasia's were diagnosed in the second trimester. MCDK affected both kidneys with a rate of $61 \%$ and when it was unilateral, it was localized in $71 \%$ of cases in the left kidney, matching the literature review.

The monitoring rate was monthly to bimonthly. The evolution was marked by stabilization of dysplasia's in $44.44 \%$ and worsening of renal dysplasia in $38.9 \%$ (the increase in the size and number of cysts in $16.7 \%$ or by the appearance of cysts in the contralateral kidney in $22.22 \%$ ) or even the decrease in the amount of amniotic fluid (16.7\%). The mortality rate was $55 \%$ for all bilateral forms as well as unilateral forms associated with a contralateral kidney malformation or other extra-renal malformations.

\section{b. Posterior urethral valves}

They represent the most severe obstructive uropathy with an annual incidence estimated at 1/5,000 male births. Antenatal diagnosis can be done in 4 out of 5 cases and can sometimes be done early at $12 \mathrm{WA}$, in our series, one case was diagnosed at $16 \mathrm{WA}$, three cases between $22 \mathrm{WA}$ and $27 \mathrm{WA}$ and four cases in the third trimester. The combination of megacystis, thickened bladder wall, dilated posterior urethra (keyhole sign), and oligohydramnios are pathognomonic for urethral valves. Without this combination of ultrasound signs, the underlying pathology of megacystis is less certain [23-25].

The ultrasound analysis does not stop at the diagnosis but it takes part in the evaluation of the prognosis, increased renal echogenicity, lack of corticomedullary differentiation, and the presence of subcortical cysts indicate renal dysplasia and are poor prognostic signs. However, due to their prenatal consequences for the upper urinary tract, which influence renal and pulmonary development, they can be of enormous clinical relevance. Up to $45 \%$ of the patients will develop renal insufficiency during childhood and prenatal diagnosis of PUVs has not improved this rate $[25,26]$.

In our series, the PUV occupied the 2nd place with a rate of $22.22 \%$ and all presented an impact on the upper apparatus: $37.50 \%$ of dysplasia, $62.50 \%$ of pyelocaliceal dilatation, we found a fetal ascites in a single fetus and a ruptured urinoma was found in another one. The mortality rate was $35 \%$ and this concerned all cases complicated by renal dysplasia, while the medium-term course was good for the rest.

\section{c. Renal Agenesis}

It corresponds to the congenital absence of the kidney, which can be bilateral or unilateral, isolated or associated with other anomalies. The bilateral form is a lethal anomaly, occurring in 0.1 to 0.3 of 1,000 live births with a higher incidence in boys, as is the case with our series [19]. Its diagnosis is most often carried out antenatal: absence of visualization of renal structures, confirmed by the absence of renal artery in color Doppler (Figure 3D, F), and a permanently empty bladder with oligoamnios from 18 SA responsible for the Potter sequence illustrated by a single case in our series (Figure 3E). It is incompatible with life like all the cases in our series.

Unilateral renal agenesis is more frequent than the bilateral form with a better prognosis [27]. The diagnosis is suggested before: an empty renal fossa with absence of ipsilateral renal artery (Figure 3G, H). In our series, a single case of unilateral left renal agenesis was found associated with other extra-renal malformations which progressed to an intrauterine death (IUD) at 32 WA confirming once again that the association with 
Hekmat Chaara et al; Sch Int J Obstet Gynec, Apr. 2021; 4(4): 114-123

other extra-renal malformations make the prognosis of CAKUT worse.

\section{d. Autosomal recessive polycystic kidney disease}

ARPK occurs with an incidence of 1 in 20,000

live births, Wilson, 2004. It is most frequently caused by a mutation in the PKHd-1 gene, but probably additional genes play a role. disease expression may vary widely within affected families. The disease is characterized by cystic dilatation of the tubules, predominantly in the medulla. The outer cortex is spared. Additionally, patients have biliary dysgenesis and hepatic fibrosis. Ultrasound scans can be normal up to 20 weeks of pregnancy. Kidneys will be markedly enlarged (+4-15 Sd) and hyperechoic without (or with reversed) corticomedullary differentiation and with a hypoechoic outer cortical. In the perinatal form, oligohydramnios as a consequence of renal failure results in lethal pulmonary hypoplasia. Children with the infantile and juvenile types develop chronic renal failure (with need for transplantation in their teens), hepatic fibrosis and portal hypertension. The recurrence rate is $25 \%$ and if the mutation is known, prenatal diagnosis can be offered [28].

In our series all our cases had oligo-anamnios, with a mortality rate of $100 \%$ : two cases were suspected early in the first trimester while the other two were described in the second trimester.

\section{e. Junction Syndrome (SJPU)}

It represents $40 \%$ of male-dominated CAKUT [18]. Antenatal ultrasound shows pyelocaliceal dilation with a pelvis with an anteroposterior diameter greater than $10 \mathrm{~mm}$ in the $3 \mathrm{rd}$ trimester of pregnancy without ureteral dilation or bladder abnormality. The evolution can take place either towards spontaneous improvement or towards worsening of the function, hence the interest in identifying by the sonographer the babies at high risk who required postnatal surgery.

Four cases of SJPU were described in our series, the evolution was towards IUD in 2 cases because of a MCDK associated with a bilateral pyélocaliciel dilation, while for the two others the evolution was good in the short term and medium term.

\section{f. Duplicated collecting system}

Duplicated collecting system concerns about $0.8 \%$ of the population. It is bilateral in $15 \%$ of cases and most often affects the female's (sex ratio $1.6 / 1$ ). The diagnosis can be done in the 2 nd half of pregnancy in front of the a large kidney which presents two sinuses separated by a parenchymal bridge and sometimes a uretero-hydronephrosis of a renal pole; The renal prognosis is mainly linked to the obstruction of the vesico-ureteral junction by an ureterocoele or an ectopic ureter, with a risk of severe dysplasia of the renal parenchyma concerned [18, 29].
In our series, we noted three cases of duplicity with a female predominance and unilateral left form. One case was hospitalized in neonatal care for prematurity and respiratory distress, who died at D23 of life, the other two had a good outcome.

\section{g. Isolated Pyelectasis}

It is a pathology for which the antenatal diagnosis remains difficult, only the Pyelectasis or the fleeting appearance of ureteral dilations during fetal urination allows the diagnosis to be made. Its diagnosis allows us to prescribe postnatal prophylaxis against urinary tract infections which can be functional or lifethreatening.

In $80 \%$ of cases, the moderate forms regress spontaneously while the severe forms require surgical treatment [28]. In our series, there were two cases of isolated unilateral pyelectasis: in one evolution was good while the second case presented a urinary tract infection complicated by jaundice.

\section{CONCLUSION}

The main goal of antenatal diagnosis of urinary tract malformations is to establish a neonatal prognosis. The methodical analysis of the parameters for all the cases in our series allowed us to draw conclusions in terms of prognosis:

- Pathologies diagnosed early generally have a genetic component with a large rate of associated extra-renal malformations and therefore a poor prognosis.

- Abnormalities in renal size, structure and echogenicity (MCDK, polycystic kidney disease) and abnormalities in number and location (renal agenesis) are affections at high risk of mortality, especially when the involvement is bilateral.

- Excretory tract abnormalities seem to have a good prognosis in their isolated form without renal dysplasia.

The amount of amniotic fluid appears to be the best indicator to assess renal function comparing to other invasive methods, oligo-anamnios is a factor of poor prognosis, on the other hand its normality has no prognostic value.

Conflicts of interest: The authors declare no conflict of interest.

Contributions from the authors: All the authors contributed to the conduct of this work. All authors also declare that they have read and approved the final version of the manuscript.

\section{State of current knowledge on the subject}

- Malformations of the urinary tract, grouped under the term CAKUT is among the most common birth defects. 
Hekmat Chaara et al; Sch Int J Obstet Gynec, Apr. 2021; 4(4): 114-123

- Antenatal diagnosis is based on obstetric ultrasound.

- The purpose of antenatal diagnosis is to make the diagnosis, look for associated malformations and establish a prognosis.

\section{Contribution of our study to knowledge}

- The number of people in our series is considerable for an African country in the absence of a referral center for antenatal diagnosis.

- The possibility of setting up the diagnosis of CAKUT while trying to predict the prognosis and to plan an appropriate postnatal care in a context of limited resources.

\section{REFERENCE}

1. Dias, T., Sairam, S., \& Kumarasiri, S. (2014). Ultrasound diagnosis of fetal renal abnormalities. Best Practice \& Research Clinical Obstetrics \& Gynaecology, 28(3), 403-415.

2. Nef, S., Neuhaus, T. J., Spartà, G., Weitz, M., Buder, K., Wisser, J., ... \& Laube, G. F. (2016). Outcome after prenatal diagnosis of congenital anomalies of the kidney and urinary tract. European journal of pediatrics, 175(5), 667676.

3. Spiro, J. E., Konrad, M., Rieger-Fackeldey, E., Masjosthusmann, K., Amler, S., Klockenbusch, W., \& Schmitz, R. (2015). Renal oligo-and anhydramnios: cause, course and outcome-a single-center study. Archives of gynecology and obstetrics, 292(2), 327-336.

4. Boussion, F., Bouderlique, C., \& Weil, D. (2011). Chapter 19: Pathology of the kidneys and urinary excretory tract; in: Prenatal diagnosis in practice. 209-222.

5. Shalaby, H., Hemida, R., Nabil, H., \& Ibrahim, M. (2015). Types and Outcome of Fetal Urinary Anomalies in Low Resource Setting Countries: A Retrospective Study. The Journal of Obstetrics and Gynecology of India. DOI 10.1007 / s13224015-0675-z.

6. Bondagji, N. (2014). Antenatal diagnosis, prevalence and outcome of congenital anomalies of the kidney and urinary tract in Saudi Arabia. Urol Ann. 6(1):36-40.

7. Uy, N., \& Reidy, K. (2015). Developmental genetics and congenital anomalies of the kidney and urinary tract. J Pediatr Genet. 5(1):51-60.

8. Capone, V. P., Morello, W., Taroni F., \& Montini, G. (2017). Genetics of congenital anomalies of the kidney and urinary tract: the current state of play. Int J Mol Sci. https://doi.org/10.3390/ijms18040796

9. Palacios Loro, M. L., Segura Ramírez, D. K., Ordo nez Álvarez, F. A., \& Santos Rodríguez, F. (2015). Congenital anomalies of the kidney and urinary tract. A vision for the pediatrician. Anales de Pediatría (Barcelona, Spain), 83(6): 442.e1442442.e5
10. Chou, C. Y., Chen, L. C., Cheong, M. L., \& Tsai, M. S. (2015). Frequency of postnatal hydronephrosis in infants with a renal anteriorposterior pelvic diameter $>4 \mathrm{~mm}$ on midtrimester ultrasound. Taiwanese Journal of Obstetrics and Gynecology, 54(5), 554-558.

11. Briard, M. L. (2007). Genetic counseling. EMC (Elsevier Masson SAS, Paris), Gynecology / Obstetrics, 5-019-A-10.

12. Jordan, I., Audra, P., \& Putet, G. (2007). Newborns to diabetic mothers. EMC (Elsevier Masson SAS, Paris), Pediatrics, 4-002-S-50.

13. Narchi, H., \& Kulaylat, N. (1997). High incidence of Down's syndrome in infants of diabetic mothers. Arch Dis Child. 77: 242-4.

14. Moore, L. L., Bradlee, M. L., Singer, M. R., Rothman, K. J., \& Milunsky, A. (2002). Chromosomal anomalies among the offspring of women with gestational diabetes. American journal of epidemiology, 155(8), 719-724.

15. Kurjak, A., Chervenak, F. A., \& Vladareanu, R. (2012). Donald School. Textbook of ultrasound in obstetrics and gynecology. Bucharest: Amaltea; 337-348.

16. CALLEN. (2017). Ultrasonography in Obstetrics and Gynecology. 6th ed. Dr Radu Vladareanu Coordinator of the Romanian edition. Hypocrate; 508-538.

17. Sabiri, N. (2013). Risk factors for congenital malformations: prospective study at the Souissi maternity hospital in Rabat, Morocco. Journal of Pediatrics and Childcare.

18. Bouty, A., Godron-Dubrasquet, A., Pelluard, F., Llanas, B., Pietrera, P., \& Dobremez, E. (2016). Congenital anomalies of renal development. EMC -Urology, 9(4):1-14.

19. Maugey-Laulom, B., \& Chateil, J. F. (2011). Antenatal diagnosis of malformative uonephropathies. EMC (Elsevier Masson SAS, Paris), Radiology and medical imaging genitourinary - gyneco-obstetric - mammary, 34760-A-25.

20. Onal, B., \& Kogan, B. A. (2006). Natural history of patients with multicystic dysplastic kidneywhat followup is needed?. The Journal of urology, 176(4), 1607-1611.

21. Van Eijk, L., Cohen- Overbeek, T. E., Den Hollander, N. S., Nijman, J. M., \& Wladimiroff, J. W. (2002). Unilateral multicystic dysplastic kidney: a combined pre- and postnatal assessment. Ultrasound in Obstetrics and Gynecology, 19(2), 180-183.

22. Kozakowski, K. A., Shah, S. M., \& Glassberg, K. I. (2010). Multicystic dysplastic kidney disease. In: Gearhart, J. P., Rink, R. C., Mouriquand, P., editors. Pediatric urology. 2nd ed. Philadelphia: Saunders Elsevier; p. 218-25.

23. Abbo, O., Bouali, O., Ballouhey, Q., Mouttalib, S., Lemandat, A., Decramer, S, Moscovici, J., \& Galinier, P. (2013). Impact of age at diagnosis on 
the long-term outcome of patients operated on for posterior urethral valves. Advances in Urology, 23, 144-149.

24. Roy, S., Colmant, C., Cordier, A. G., \& Sénat, M. V. (2015). Contribution of ultrasound signs for the prenatal diagnosis of posterior urethral valves: Experience of 3years at the maternity of the Bicetre Hospital. Journal de gynecologie, obstetrique et biologie de la reproduction, 45(5), 478-483.

25. Perks, A. E., Mac Neily, A. E., \& Blair, G. K. (2002). Posterior urethral valves. J Pediatr Surg, 37(7):1105-7.

26. Lopez Pereira, P., Espinosa, L, Martinez Urrutina, M. J., Lobato, R., Navarro, M., \& Jaureguizar, E.
(2003). Posterior urethral valves: prognostic factors. BJU Int, 91: 687-90.

27. Kalfa, N., Veyrac, C., Dubois, C., Morin, D., Lopez, C., \& Averous, M. (2009). Congenital malformations of the kidney. EMC (Elsevier Masson SAS, Paris), Urology, 18-125-A-10.

28. Driessen, M., Chéreau, E., Aubry, M. C., VibertGuigue C., Ruano R., \& Dommergues, M. (2014). Fetal urogenital anomalies. EMC - Obstetrics / Gynecology, 9(1):1-20.

29. Dariane, C., Chartier-Kastler, E., \& Rouprêt, M. (2015). Congenital malformations of the ureter in adults. EMC - Urology, 8(3):1-10. 